\title{
Kinetic Infernal Modes for Wendelstein 7-X-like $\iota$-profiles
}

\author{
Alessandro Zocco, Alexey Mishchenko, Axel Könies \\ Max-Planck-Institut für Plasmaphysik, 17489, Greifswald, Germany
}

(Received 22 October 2019)

We show analytically that for $\iota$-profiles similar to the one of the Wendelstein 7-X stellarator, where $\iota$ is the rotational transform of the equilibrium magnetic field, a highly conducting toroidal plasma is unstable to kinetically mediated pressure-driven long-wavelength reconnecting modes, of the infernal type. The modes are destabilized either by the electron temperature gradient or by a small amount of current, depending on how far from unity is the average value of $\iota$, which is assumed to be slowly varying. We argue that, for W7-X, a broad mode with toroidal and poloidal mode numbers $(n, m)=(1,1)$ can be destabilized due to the strong geometric side-band coupling of the resonant kinetic electron response at locations where $\iota$ is rational for harmonics that belong to the mode family of the $(n, m)=(1,1)$ mode itself. In many regimes, the growth rate is insensitive to the plasma density, thus it is likely to persist in high performance W7-X discharges. For a peaked electron temperature, with a maximum of $T_{e}=5 \mathrm{keV}$, larger than the ion temperature, $T_{i}=2.5 \mathrm{keV}$, and a density $n_{0}=10^{19} \mathrm{~m}^{-3}$, instability is found in regimes which show plasma sawtooth activity, with growth rates of the order of tens of kiloHertz. Frequencies are either electron diamagnetic or of the ideal magnetohydrodynamic type, but sub-Alfvenic. The kinetic infernal mode is thus a good candidate for the explanation of sawtooth oscillations in present-day stellarators and poses a new challenge to the problem of stellarator reactors optimization.

\section{Introduction}

The stellarator Wendelstein 7-X (Klinger et al. 2013, 2017) has been carefully designed to reach long, steady discharges and avoid the operational issues of previous stellarators, putting emphasis on good neoclassical transport properties. In this regard, the neoclassical theory of transport has been, and still is, the cornerstone in the optimization of confinement properties (Dinklage et al. 2018). Regarding plasma stability, on the other hand, magnetohydrodynamics has always been the "non plus ultra" in stellarator design, and good MHD behaviour is always sought in their optimization, as it was in W7-X (Nührenberg \& Zille 1987).

A particular kind of instability that is usually believed to be absent in modern stellarators are reconnecting modes. Indeed, magnetic reconnection is often thought to be solely driven by the gradient of the equilibrium current density. Since in carefully optimised stellarators there is virtually no, or very little, equilibrium current (even if magnetic islands might be present and used by design), magnetic reconnection instabilities are usually not seen as an issue of concern, especially for quasi-isodynamic and quasi-poloidally symmetric stellarators. Novel experimental evidence challenges this simplistic view, however.

It is now an accepted fact that W7-X shows sawtooth oscillations similar to those first observed in tokamaks (Zanini, M. et al. 2019). Since sawteeth are generally understood invoking magnetic reconnection (Hastie 1997; Porcelli et al. 1996), a great effort was put in the evaluation of the effect of the electron current drive in the modification of the 


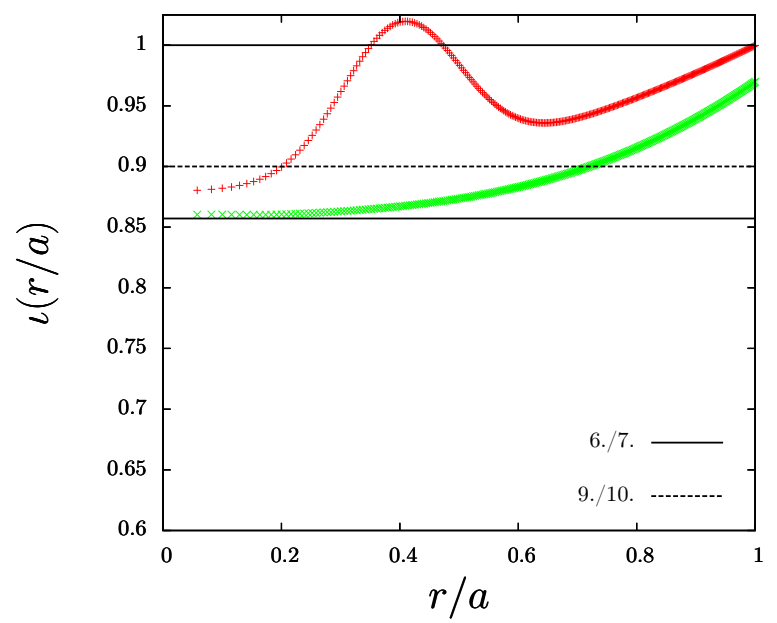

Figure 1. Radial profile of 2 rotational transforms in W7-X, vacuum-like (green) and ECCD distorted (red). Locations of resonances of $n_{0} / m_{0}=1$, and the side-band of the harmonics $n_{1} / m_{1}=6 / 6$, and $n_{2} / m_{2}=9 / 9$, that is $n_{1} /\left(m_{1}+1\right)$, and $n_{2} /\left(m_{2}+1\right)$. Notice that $n_{1} / m_{1}$ and $n_{2} / m_{2}$ belong to the same mode-family of $n_{0} / m_{0}=1$, and thus couple to it (Nührenberg 1996).

$\iota$-profile, to verify whether an ideal kink-like MHD instability would manifest itself just before current crashes, for $\iota$-profiles that posses resonant locations, that is $\iota=n / m$, with $n$ and $m$ integers. A typical $\iota$ profile for such discharges is in Fig. 1.

Extensive numerical studies have excluded the presence of ideal MHD instabilities for such $\iota$-profiles (Könies \& Nührenberg 2019). While this is a positive sign regarding the MHD properties of W7-X, instabilities enabled by non-ideal effects cannot be completely ruled out when it comes to analyzing the true plasma behaviour beyond its basic designbased properties. We are therefore motivated to consider magnetic reconnection as a physical mechanism that can cause sawtooth-like oscillations in W7-X.

In tokamaks, magnetic reconnection events that are associated with sawteeth generate a displacement of a whole plasma "column", enclosed by a surface of constant magnetic flux, within a larger flux surface.When magnetic flux surfaces get close enough and small non-ideal scales start to matter, magnetic reconnection occurs. For a resistive plasma, the typical scale governing the destabilization of a current-driven reconnection instability is (Ara et al. 1978; Porcelli 1987; Zocco \& Schekochihin 2011) $\delta_{\eta} \sim\left(\nu_{e i} d_{e}^{2} /\left(v_{A} a\right)\right)^{2 / 3} a^{2} / \rho_{s}$, where $v_{A}=B / \sqrt{4 \pi m_{i} n_{0}}$ is the Alfven speed, $\nu_{e i}$ is the electron-ion collision frequency, $d_{e}=c / \omega_{p e}$ is the electron skin depth, with $\omega_{p e}$ the electron plasma frequency, $\rho_{s}=\left(T_{e} / T_{i}\right)^{1 / 2} \rho_{i}$ is the sonic ion Larmor radius, $T_{e}$ and $T_{i}$ are the electron and ion temperatures, $\rho_{i}$ the ion Larmor radius, and $a$ is a macroscopic scale, taken to be the average minor radius. For typical W7-X values, $B=2.510^{4} G, T_{i}=2 \mathrm{keV}$, $T_{e}=5 \mathrm{keV}(\approx 2 \mathrm{keV}$ at a resonant location $), n_{0}=10^{13} \mathrm{~cm}^{-3}$, and $a=50 \mathrm{~cm}$, we find $\delta_{\eta} \approx 5.5 \times 10^{-3} \mathrm{~cm}$. This scale is much smaller than the ion Larmor radius $\rho_{i} \approx 0.2 \mathrm{~cm}$, and, most importantly, the electron skin depth $d_{e} \approx 0.17 \mathrm{~cm}$. We conclude that, owing to its high temperature, collisional current-driven (kink) modes are negligible in W7-X, at least in the plasma core. In the plasma edge, where the temperature is lower, we might expect resistivity to be more effective, but we are not concerned with this problem here.

In order to develop our intuition on how magnetic reconnection behaves in W7-X relevant regimes, we construct a model $\iota$-profile that allows us to discriminate between pressure-driven and current-driven ideal instabilities, the former being also important due 
to the small global shear. The ideal MHD analysis is carried out in cylindrical geometry. While we are aware of the geometric limitations of such a model, the interplay between ideal and kinetic instabilities for W7-X-like $\iota$-profiles is by no means obvious, not even in a cylinder, and will be clarified in this work.

The toroidal analysis of the pressure-driven ultra-flat- $q$ (infernal)(Hastie \& Hender 1988; Waelbroeck \& Hazeltine 1988) mode of Brunetti et al. (2014) is then extended to kinetic regimes, thus providing new insight on the possibility of driving kinetic magnetic reconnection in W7-X. Our main findings are: in W7-X $\iota$-profiles, in a cylinder, kink-like instabilities are mostly pressure-driven, owing to the small global shear; the presence of an $\iota(r)=1$ resonant location is crucial in determining the instability of either pressuredriven kink modes or pressure-driven infernal modes, but in fact the character of the instability is a hybrid of the two; in toroidal geometry, when ideal infernal modes are stable, kinetic effects can destabilize a kinetic infernal mode, even in the absence of an $\iota(r)=1$ resonance, when the plasma is ideally stable, and side-band resonances of harmonics of the $(m, n)=(1,1)$ mode can be excited.

\section{Setting and ideal MHD properties}

As it will be seen shortly, for ideal instabilities that cause the displacement of the plasma column, the pressure gradient competes with the current drive for $\beta \sim \epsilon^{2}$, where $\epsilon$ is the inverse aspect ratio, and can be neglected only for ultra-low $\beta$. However, for most fusion experiments, this is not the case. It is also crucial to know that, while the information about the ordering of $\beta$ is useful, the effectiveness of the current drive for these instabilities, when $\iota$ is mostly flat, is measured by the distance of the values of the rotational transform from unity. It turns out that this fact introduces a subtlety in the classification of pressure-driven ideal modes, which we would like to recall here. Depending on the $\iota$-profile, we can have two types of such modes. For a strongly varying $\iota=q^{-1}$, that is when $\hat{s}=r q^{\prime}(r) / q(r)$ is significantly larger than unity, in the presence of a rational surface $\iota=1$, we have the pressure-driven ideal kink mode (Newcomb 1960; Goedbloed \& Hagebeuk 1972; Rosenbluth et al. 1973), with growth rate $\gamma \sim \beta v_{A} / a \sim$ $\sqrt{n_{0} T} v_{t h i} /(B a)$, thus scaling with the square root of density. For a flat $\iota$-profile [see the "Tokamak" case in Fig. 1], we have the ultra-flat- $q$ internal (or infernal) mode (Hastie \& Hender 1988; Waelbroeck \& Hazeltine 1988) with growth rate $\gamma \sim \sqrt{\beta} v_{A} / a \sim v_{t h i} / a$, thus showing no scaling with density. The latter result is derived for a $q=\iota^{-1}$ profile which is constant and larger than (but close to) 1 for $0 \leqslant r \leqslant r_{*}$, and increasing for $r_{*} \leqslant r \leqslant a$. This is the opposite of a W7-X profile, where $q$ decreases for $r_{*} \leqslant r \leqslant a$, and we can have, as we will show, hybrid modes that share features common to the kink and the infernal mode. Let us then introduce the following $q$-profile model

$$
q(r)=-\frac{q_{0}-1}{r_{s}-r_{*}} r+\frac{q_{0} r_{s}-r_{*}}{r_{s}-r_{*}}, \text { for } r>r_{*},
$$

and proceed with our initial cylidrical MHD analysis. Here $q_{0}$ is a constant larger than unity, $r_{s}$ is so that $q\left(r_{s}\right)=1$, (the radial location at which resonance occurs) $r_{*}$ is so that $q(r)=q_{0}$ for $0 \leqslant r \leqslant r_{*}$, (the radial location up to which $q$ is constant) and $0 \leqslant r \leqslant a$. Notice that in the following analysis we will use $q$ rather than $\iota$, for historic reasons.

For a radial plasma displacement $\xi(r) \cos \left(m \theta+k_{z} z\right)$, the equation of the normal mode is (Newcomb 1960)

$$
\frac{d}{d r}\left[4 \pi \rho \gamma^{2}+(\mathbf{k} \cdot \mathbf{B})^{2}\right] r^{3} \frac{d \xi}{d r}=g_{1} \xi
$$




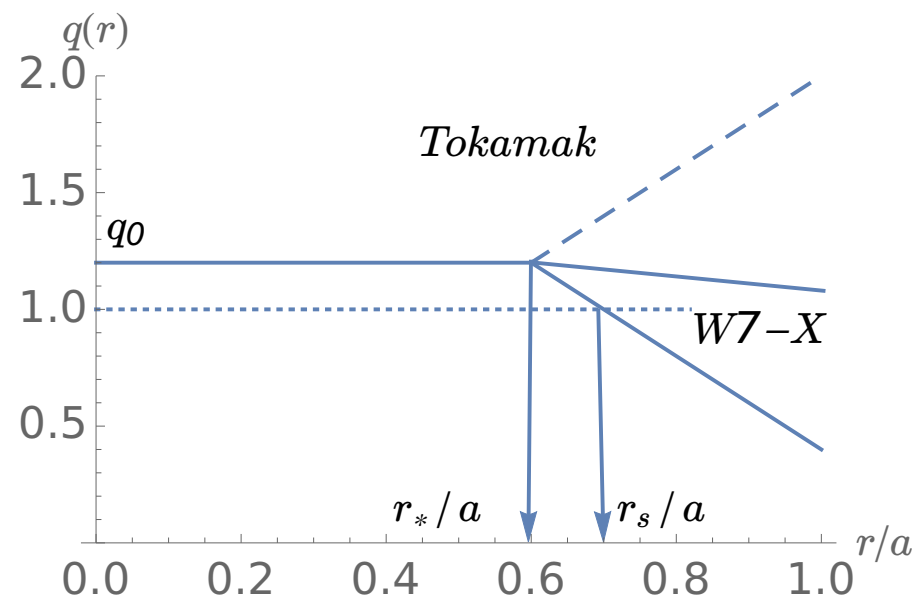

Figure 2. Example of the model $q$-profile of our analysis. The tokamak case is compared to two W7-X cases, with and without $q=1$ resonant surface.

where $\gamma$ is the growth rate, $\rho$ the plasma density,

$$
g_{1}=\frac{r^{2}}{R^{2}}\left\{8 \pi \frac{d p}{d r}+2 \frac{B_{z}^{2}}{R^{2}} r\left(1-\frac{1}{q}\right)+r(\mathbf{k} \cdot \mathbf{B})^{2}\right\},
$$

$R$ is the length of the cylinder, we are considering $m=n=1$, and we are taking $r \ll R$. It is easy to show that the tearing mode stability parameter is (Ara et al. 1978)

$$
r_{s} \Delta^{\prime}=\frac{\left[B_{\theta}\left(r_{s}\right) r_{r} q^{\prime}\left(r_{s}\right)\right]^{2}}{\frac{1}{8} \int_{0}^{r_{s}} g_{1} d r} .
$$

The system is tearing-mode stable for $\Delta^{\prime}<0$, and tearing-mode unstable for $\Delta^{\prime}>\Delta_{\text {crit }}^{\prime}$, where $\Delta_{\text {crit }}^{\prime}$ has been recently evaluated by Connor et al. (2019).

Since for $0 \leqslant r \leqslant r_{*} q$ is constant, we have $B_{\theta}(r)=(r / R) B_{z} / q_{0}$, where $B_{z}$ is constant. Furthermore, $k_{\theta}=-1 / r$, and Eq. (2.2) becomes

$$
\frac{d^{2} \xi}{d r^{2}}+\frac{3}{r} \frac{d \xi}{d r}=\frac{1}{\hat{\gamma}^{2}+\frac{\delta q^{2}}{q_{0}^{2}}}\left(\frac{1}{r} \frac{d \beta}{d r}+\frac{2}{R^{2}} \frac{\delta q}{q_{0}}\right) \xi,
$$

where $\beta=8 \pi p(r) / B_{z}^{2}$, and $\delta q=q_{0}-1>0$. This equation is particularly simple to solve for parabolic pressure profiles. We thus take $p(r)=p_{0}\left(1-r^{2} / L_{p}^{2}\right)$, with $p_{0}=n_{0}\left(T_{e}+T_{i}\right)$, and find $\beta^{\prime}=-18 \pi p_{0} r / L_{p}^{2} B_{z}^{2}$. We consider the three possible scenarios proposed in Fig. 1. For a tokamak-like $q$-profile $\left(\hat{s}>0\right.$ for $r_{*}<r<a$ ), the result of Goedbloed \& Hagebeuk (1972), reported by Waelbroeck \& Hazeltine (1988), is found, and an unstable pressure driven mode is derived by applying the boundary condition $\xi_{e x t}\left(r_{*}\right)=0$, where $\xi_{\text {ext }}(r)=$ $(a / r) J_{1}\left(\kappa r / L_{p}\right)$, with $\kappa^{2}=2\left(\hat{\gamma}^{2}+\delta q^{2} / q_{0}^{2}\right)^{-1}\left[\beta_{0}-\left(\delta q^{2} / q_{0}^{2}\right) L_{p}^{2} / R^{2}\right], \beta_{0}=8 \pi p_{0} / B_{z}^{2}$, and $\hat{\gamma}=\gamma /\left(v_{A} / R\right)$. For the W7-X-like $\iota-$ profile $(\hat{s}<0)$, we use the solution just found as a boundary condition at $r=r_{*}$ for the solution valid at $r=r_{s}$. In the case in which $q=1$ at $r_{s}$, for $r \sim r_{s}$, one finds (Rosenbluth et al. 1973) $\xi_{\text {inner }}(r)=2^{-1} \xi_{a}\left[1-(2 / \pi) \arctan \left(r / r_{s}-\right.\right.$ $\left.1)|\hat{s}| /\left(q_{0} \hat{\gamma}\right)\right]$, where $\xi_{a}$ is a constant. By imposing

$$
\left(\xi_{\text {inner }}^{\prime} / \xi_{\text {inner }}\right)_{r_{*}}=\left(\xi_{\text {ext }}^{\prime} / \xi_{\text {ext }}\right)_{r_{*}},
$$

we obtain an eigenvalue equation for an eigenfunction that has structure at $r=r_{s}$, but matches smoothly onto an infernal-like solution for $r<r_{s}$ (see Fig. 3). 
In order to verify the structure of the eigenfunctions in Fig. 3, we solve numerically Eq. (2.2) with a shooting method, by introducing the smoothed $q$-profile, matching $q_{0}$ to the $q(r)$ of Eq. (2.1),

$$
q_{S}(r)=\frac{q_{1}(r)+q_{2}(r)}{q_{1}(0)+q_{2}(0)} q_{1}(0),
$$

where $q_{1}(r)=\left(q_{0} / \pi\right)\left[\pi / 2-\arctan \left(\left(r-r_{*}\right) / \delta r\right)\right], q_{2}(r)=(q(r) / \pi)[\pi / 2+\arctan ((r-$ $\left.\left.\left.r_{*}\right) / \delta r\right)\right]$, and $\delta r \ll 1$ is a constant to be suitably chosen. For $q_{0}=1.1, r_{*} / a=0.6$, $r_{s} / a=0.65, R / a=3, \beta_{0}=(0.04,0.05)$, and $\delta r=10^{-3}$ we find two unstable sub-Alfvenic modes $\hat{\gamma}=(1.17,1.58) \times 10^{-2}$. The plasma beta is therefore destabilizing, like the analytic dispersion relation, Eq. (2.6), predicts. The numerical eigenvalues are somewhat smaller than the analytical ones, due to the fact that the shooting code actually solves for the full cylindrical problem for finite $k_{z}=n / R$, and $r / R$. We are not interested in the search of the asymptotic limits in which the two methods give quantitative agreement. What is striking is the structure of the eigenfunctions [see Fig. (4)], which indeed are of a hybrid type between those of the kink and infernal mode, for a W7-X-like $q$-profile. This information will be useful in future W7-X investigations where the full geometry will be considered. We now study the dependence of the growth rate with density, which we scan for $B_{z}=2.5 \times 10^{4} G, R=3, a=1, T_{i}=2 \mathrm{keV}$, and $T_{e}=5 \mathrm{keV}$. Here $r_{*} / a=0.6$, and $r_{s} / a=0.65$ for "Tok-like" $q$-profiles, while $r_{*} / a=0.55$, and $r_{s} / a=0.65$ for the W7-X-like $q$-profiles. In the latter case $r_{*} / a$ is chosen not too close to $r_{s} / a$ in order to keep $q(a)>1 / 2$. While a tokamak-like $q$-profile with no resonant $q=1$ surface only shows an infernal mode (with growth rate nearly independent of $\beta$ for large density and a sharp transition at marginality) [see "Tok-like" in Fig. (5)], the "Tok-like" $q$-profile with a resonant $q=1$ surface shows a persistent (current-driven) instability even for small pressure drives. On the other hand, the W7-X-like $q$-profile shows an infernal mode where instability is allowed below the critical $\beta$ of the Tok-like $q-$ profile with no resonant $q=1$.

Of the three $q$-profiles proposed in Fig 1, the most interesting case, from a kinetic perspective, is the one for which there is no $q=1$ surface by design, and $\hat{s}<0$. This is the one least prone to ideal MHD instabilities, but, as we shall see, kinetics can play a major role in driving magnetic reconnection.

\section{Kinetic Theory}

We now present a kinetic theory for the W7-X $\iota$-profile of the second type, that is with no $\iota=1$ resonance. Here we consider the resonant response of the electron and ions, by implementing in Brunetti's theory (Brunetti et al. 2014) the layer response of Connor et al. (2019), and of Ref. (Zocco et al. 2015). The analysis is valid in toroidal geometry, but limited to axisymmetric shifted circular flux surfaces. This is still relevant if we focus on the identification of the kinetic effects that will change the known viscous-resisitve picture (Porcelli 1987; Brunetti et al. 2014) at the resonant locations. We therefore study the ideal plasma response of the $(m, n)$ mode for $0 \leqslant r \leqslant r_{*}$, coupled to the resonant response of the side-band $(m+1, n)$ at $r=r_{s}$. We will show that, for $q\left(r_{s}\right)=7 / 6$, and $q\left(r_{s}\right)=10 / 9$, the $(m, n)=(6,6)$ and $(m, n)=(9,9)$ can be kinetically excited. In W7$\mathrm{X}$, we expect this kinetic mechanism to excite the $(m, n)=(1,1)$ mode as well, due to the strong geometric coupling of the harmonics of the same mode families (Nührenberg 1996).

We consider the infernal mode dispersion relation (Brunetti et al. 2014, 2015) in the 


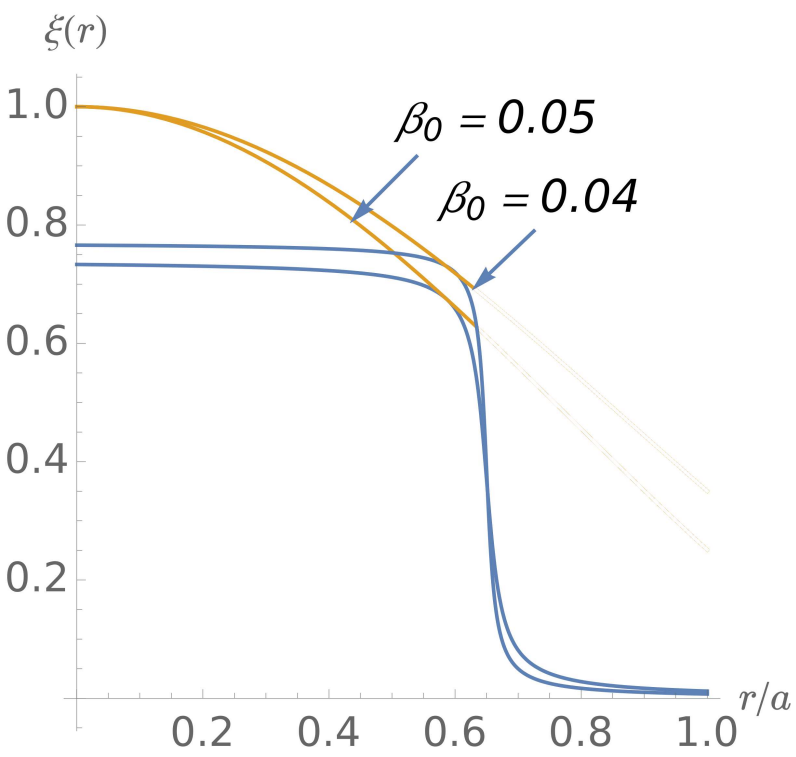

FIgURE 3. Inner layer (nearly constant for $r \lesssim 0.6 a$ ) and MHD solutions for W7-X-like $\iota$-profile, with $\iota=1$ at a given $r=r_{s}$, with $r_{*}<r_{s}<a$. Here $R=3 a, L_{p}=a, r_{*}=0.6, r_{s}=0.65$, $q_{0}=1.1$. For $\beta_{0}=0.04, \hat{\gamma}=1.88 \times 10^{-2}$, for $\beta_{0}=0.05, \hat{\gamma}=3.27 \times 10^{-2}$, where $\beta_{0}$ and $\hat{\gamma}$ are defined in the text, and $\hat{\gamma}$ is the solution of Eq. (2.6).

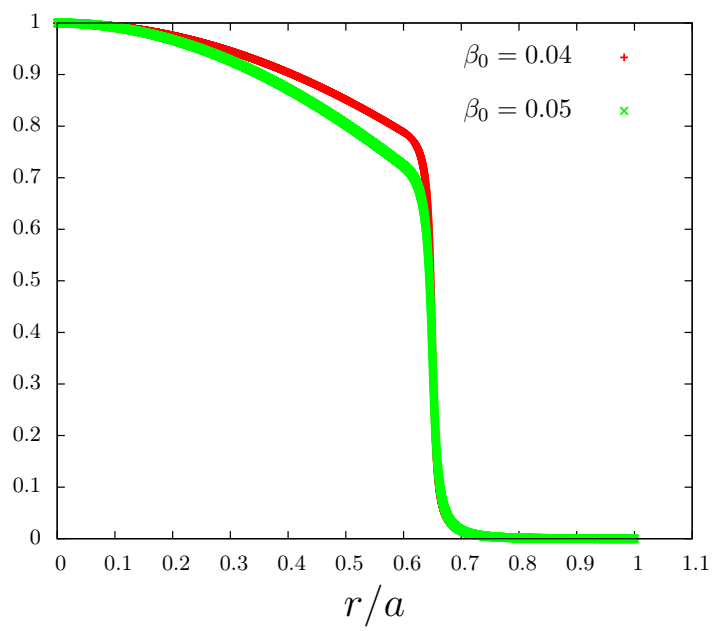

FIGURE 4. Eigenfunctions for W7-X-like $q$-profiles with resonant surface as evaluated from a shooting code. The hybrid nature between infernal and kink mode is evident and agrees with the eigenfunction of Fig. 3, predicted by the analytical calculation.

flat density limit, which is often relevant in W7-X

$$
\frac{\omega^{2}}{\omega_{A}^{2}}-\frac{n^{2}}{1+2 q_{0}^{2}}\left(\frac{\delta q^{2}}{q_{0}^{2}}+\beta_{p}^{2} G_{0}\right)=-\frac{\beta_{p}^{2}}{r_{s} \Delta^{\prime}} G_{0}\left(B_{0}-A_{0}\right)
$$

where $\beta_{p}=p_{0} q_{0}^{2} /\left(B_{z}^{2} \epsilon_{*}^{2}\right)\left(a / L_{p}\right)^{4}$, and $\Delta^{\prime}$ is the tearing mode stability parameter of the $m+1$ mode at the $q=m+1 / n$ location expressed in terms of layer quantities. This will be derived from the kinetic drift-tearing mode dispersion relation. The $\Delta_{m+1}^{\prime}$ evaluated from 


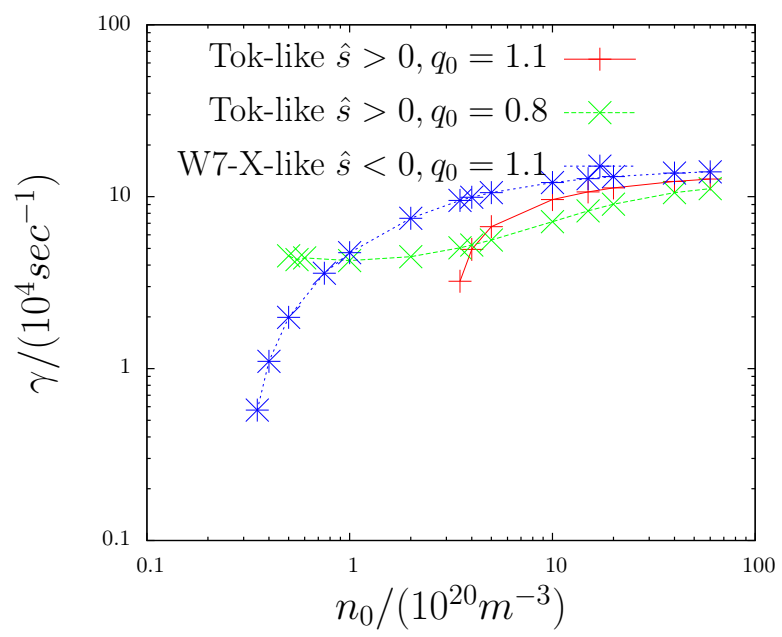

Figure 5. Eigenvalues for three types of $q$-profiles with and without resonant surface as evaluated from a shooting code as a function of the plasma density. Here $r_{*} / a=0.6$, and $r_{s} / a=0.65$ for "Tok-like" $q$-profiles, while $r_{*} / a=0.55$, and $r_{s} / a=0.65$ for the W7-X-like $q$-profiles. In the latter case $r_{*} / a$ is chosen not too close to $r_{s} / a$ in order to keep $q(a)>1 / 2$. Other parameters are $B_{z}=2.5 \times 10^{4} G, R=3, a=1, T_{i}=2 \mathrm{keV}$, and $T_{e}=5 \mathrm{keV}$.

the ideal limit of the equation for the $m+1$ harmonic drops from the picture since we are considering the $r_{s} \Delta^{\prime} \gg 1$ limit of the result derived by Brunetti et al. In this case $\Delta_{m+1}^{\prime}$ cancels exactly and, in Eq. (3.1), $\Delta^{\prime}$ is a quantity solely determined by layer physics. Indeed, resistive infernal modes are observed to be unstable close to ideal marginality, $\delta q^{2} / q_{0}^{2} \approx \beta_{p}^{2}\left|G_{0}\right|$, with $G_{0}<0$, even if $\Delta_{m+1}^{\prime}<0$ (Brunetti et al. 2015). We then see that the $\Delta^{\prime} \gg 1$ limit is appropriate, since layer effects are important only for $\Delta^{\prime} \delta \sim 1$, where $\delta$ is the width of the linear reconnecting mode, and $\delta / r_{s} \ll 1$. The quantities $G_{0}=\epsilon_{*}^{2} \hat{s}_{*} /[(\hat{s}-2)(m+1)(m+2)]$, and $B_{0}-A_{0}=8(m+1)^{3} \bar{C} /\left[C_{1}^{2}+2(m+1) C_{1}\right]$, contain the ideal MHD information. All the quantities with an asterisk are evaluated at $r_{*}$. Hence $(m+1)(m+2) G_{0}=\epsilon_{*}^{2} \hat{s}_{*} /\left(\hat{s}_{*}-2\right) \approx-\epsilon_{*}^{2} \hat{s}_{*} / 2>0$, for $\left|\hat{s}_{*}\right| \ll 1$ and $\hat{s}_{*}<0$, which is our case. Notice that if we set to zero the term that couples kinetics to the infernal mode, via $\Delta^{\prime}$, on the RHS of Eq. 3.1, for $G_{0}>0$ the system is ideally stable. This is consistent with the ideal MHD analysis of the monotonic vacuum-like $\iota$-profiles of W7-X, which show no ideal instability. We will then consider $G_{0}>0$ always. The sign of the quantity $B_{0}-A_{0}$ depends on the value of $\delta q$. Indeed, since $\bar{C}=\Gamma^{2}(1-\Lambda)\left(r_{*} / r_{s}\right)^{2(m+1)}>0$, for any $\Lambda$ real (which we do not need to define here), and $C_{1}=(m+1) r_{*} \iota_{*}^{\prime} /\left[(m+1) \iota_{*}-n\right]$, we find that $B_{0}-A_{0}>0$ if $\delta q<\left(1+|\hat{s}| / 2 q_{0}\right) / n$. Thus, when all kinetic effects are negligible, and the quantity $\Delta^{\prime}$ is real, Eq. (3.1) implies that what would give an unstable resistive mode at $r=r_{s}\left(\Delta^{\prime}>0\right)$ has a destabilizing effect on the ideally stable infernal mode $\left(G_{0}>0\right)$ for $B_{0}-A_{0}>0$, that is, when $\delta q<\left(1+|\hat{s}| / 2 q_{0}\right) / n$. Vice versa, what would give an unstable resistive mode $\left(\Delta^{\prime}>0\right)$ has a stabilising effect on the infernal mode if $\delta q>\left(1+|\hat{s}| / 2 q_{0}\right) / n$. Notice that, since in the derivation of Eq. (3.1) $\delta q \sim \beta_{0} \ll 1$ is assumed, the condition $\delta q>\left(1+|\hat{s}| / 2 q_{0}\right) / n$ would always break the underlying ordering of $\delta q$ for mode numbers of physical interest, $n \ll \beta_{0}^{-1}$. We therefore exclude the case $\delta q>\left(1+|\hat{s}| / 2 q_{0}\right) / n$ from our analysis. In the kinetic case, $\Delta^{\prime}$ is a complex quantity, but the result must be kept consistent with our fluid considerations, for negligibly small kinetic effects.

We now replace in the dispersion relation the $\Delta^{\prime}$ written in terms of the layer quantities 
at the resonant location(Zocco et al. 2015), which is evaluated from

$$
\frac{\delta}{\rho_{i}} B(\hat{\omega})=\frac{2}{\pi} I_{e} \hat{\omega}^{2} C(\hat{\omega}),
$$

where

$$
\begin{gathered}
B(\hat{\omega})=\frac{\Delta^{\prime} \rho_{i}}{\pi \hat{\beta}_{T}}-\hat{\omega}^{2} \frac{Z / \tau}{[Z / \tau+1]^{2}} \frac{1}{\sqrt{\pi}} \log \frac{\rho_{i}}{\delta}+\hat{\omega}^{2} \bar{I}(\tau) \\
\equiv \frac{\rho_{i}}{\pi \hat{\beta}_{T}}\left[\Delta^{\prime}-\left(\Delta_{F L R}^{\prime}+\Delta_{\text {diam }}^{\prime}\right)\right] \\
C(\hat{\omega})=1-\frac{\hat{\beta}_{T} \Delta^{\prime} \rho_{i}}{\pi} \hat{\omega}^{2} I(\tau), \\
I(\tau)=\int_{0}^{\infty} d q\left\{\frac{F}{G}-\frac{Z / \tau}{Z / \tau+1}+\frac{Z / \tau}{\sqrt{\pi}(Z / \tau+1)^{2}} \frac{1}{1+q}\right\},
\end{gathered}
$$

with $\bar{I}(\tau)=\int_{0}^{\infty} d q F /\left(q^{2} G\right), F(q)=(Z / \tau)\left(\Gamma_{0}(q)-1\right), G(q)=F(q)-1, \Gamma_{0}(q)=$ $I_{0}(q / 2) \exp \left(-q^{2} / 2\right)$, where $I_{0}$ is the modified Bessel function, $\hat{\beta}_{T}=0.5 \beta_{e} L_{s}^{2} / L_{T}^{2}$, $\hat{\omega}=$ $\omega / \omega_{T}, \omega_{T}=\omega_{* e} L_{n} / L_{T}, \omega_{* e}=2^{-1} m v_{t h e} \rho_{e} /\left(L_{n} a\right)$ is a local approximation of the electron diamagnetic frequency, with $\rho_{e}$ the electron Larmor radius, $L_{n}^{-1}=n_{0}^{-1} d n_{0} / d r$, $L_{s}^{-1}=q^{-1} d q / d r \approx \hat{s} / a, \tau=T_{i} / T_{e}$, and $Z$ the ion charge number. Depending on the magnitude of $\Delta^{\prime}$, different kinetic effects are involved at the resonant location. Before Eq. (3.3) applies, for marginally stable kinetic drift-tearing modes, the relevant expression for the critical $\Delta_{c r i t}^{\prime}$, which takes into account ion Landau damping, gives (Connor et al. 2019)

$$
\frac{1}{\Delta_{c r i t}^{\prime}}=2 \frac{\rho_{i}}{\beta_{i}}\left(\frac{\omega_{* e}}{\omega} \frac{L_{n}}{L_{s}}\right)^{1 / 2} \frac{1}{I(a)},
$$

where $a=\left(\omega / \omega_{* e}\right)\left(L_{s} / 2 L_{n}\right)$. The quantity $I(a)$ is complex, and its imaginary part is negative (Connor et al. 2019). If one now considers an ideally stable infernal mode, $G_{0}>0$, from Eq. (3.1) we find, to leading order, two stable MHD modes

$$
\omega= \pm(n / \sqrt{3})\left(\delta q^{2} / q_{0}^{2}+\beta_{p}^{2} G_{0}\right)^{1 / 2} \equiv \omega_{ \pm} .
$$

We choose the frequency that connects to the drift-tearing mode for larger $\Delta^{\prime}$. This gives a mode rotating in the electron diamagnetic direction $\omega \omega_{* e}>0$, with $\omega_{* e}>0$. Then, since $\Im[I(a)]<0$, we see that ion Landau damping on drift-tearing modes has a stabilizing effect on the infernal mode. In toroidal geometry, the ion Landau damping effect will compete with the critical $\Delta_{G}^{\prime}$ evaluated by Glasser et al., set by geometry (Glasser et al. 1975). Which one prevails is a question that bears on the marginal stability properties of the $m+1$ mode, and this is not of great importance in our discussion.

For very large tearing stability parameters, $\Delta^{\prime} \rho_{i} \sim \beta^{-1} L_{T}^{2} / L_{s}^{2}$, the drift-tearing mode enters the ion-kinetic regime (Pegoraro et al. 1989), and couples to a kinetic Alfven wave (Connor et al. 2012). In this case, Eq. (3.2) is dominated by its RHS, and we have

$$
\Delta^{\prime} \approx \Delta_{K I}^{\prime}=\frac{1}{\rho_{i}} \frac{2 \pi L_{T}^{2}}{\beta_{e} L_{s}^{2}} \frac{\omega_{T}^{2}}{\omega^{2} I(\tau)},
$$

where $I(\tau)$ is a real positive quantity (Zocco et al. 2015), and we are considering the flat density limit, in accordance with the experimental profiles. By replacing Eq. (3.6) in (3.1), one sees that ion Larmor radius kinetic effects reduce the growth rate of an 
unstable ideal infernal mode $\left(\beta_{p}^{2} G_{0}>\delta q^{2}\right.$, with $\left.G_{0}<0\right)$, but cannot destabilize the ideally stable one, $G_{0}>0$.

When the tearing mode parameter is above the critical value of Antonsen \& Coppi (1981), $\Delta_{c r i t, A C}^{\prime}=\Delta_{F L R}^{\prime}+\Delta_{\text {diam }}^{\prime}$, but below $\Delta_{K I}^{\prime}$, the layer physics is dominated by electron dynamics. Then (Coppi et al. 1979; Drake 1978; Zocco et al. 2015)

$$
\Delta^{\prime} \approx \Delta_{D T}^{\prime}=\frac{\beta_{e}}{\delta} \frac{L_{s}^{2}}{L_{T}^{2}} I_{e}\left(\frac{\omega}{\omega_{T}}\right) \frac{\omega^{2}}{\omega_{T}^{2}},
$$

where

$$
\begin{aligned}
I_{e} & \approx \frac{1}{2} \int_{0}^{\infty} d s\left[\frac{1}{s} Z^{\prime}\left(\frac{1}{s}\right)+\frac{1}{2 \hat{\omega}} Z^{\prime \prime}\left(\frac{1}{s}\right)\right] \\
& =-i \frac{\sqrt{\pi}}{2}\left(1-\frac{1}{2 \hat{\omega}}\right),
\end{aligned}
$$

and $Z$ is the plasma dispersion function. Notice that in Eq. (3.7) there is no dependence on the sign of $\hat{s}$. We now replace Eq. (3.7) in Eq. (3.1), neglect numerical factors, and obtain

$$
\begin{aligned}
& \frac{\omega}{v_{\text {the }} / a} I_{e}(\omega)\left[\frac{\omega^{2}}{\omega_{A}^{2}}-\frac{1}{3}\left(\frac{\delta q^{2}}{q_{0}^{2}}+\beta_{p}^{2} G_{0}\right)\right]= \\
& -|\hat{s}|(m+1) \beta_{p}^{2} G_{0}\left(B_{0}-A_{0}\right) \frac{d_{e}^{2}}{a^{2}},
\end{aligned}
$$

where we used $\delta=\omega /\left((m+1) v_{\text {the }} / 2 a\right) a / \hat{s}$. Equation (3.9) describes the destabilisation of the kinetic infernal mode (KIM). It tells us that, for negligible magnetic reconnection $\left(d_{e} \rightarrow 0\right)$, we have two decoupled branches: a stable ideal MHD mode, with frequency $\omega_{0}= \pm \omega_{ \pm}$; and a stable drift-tearing mode, with frequency $\omega_{D T}=\omega_{T} / 2$.

Both modes can be destabilized resonantly. We show this by solving the KIM mode equation (3.9) in a subsidiary low $d_{e} / a$ expansion, for infinitesimally small drive. We seek a root $\omega=\omega_{0}+\delta \omega$. To leading order we have

$$
\left(\omega_{0}-\frac{\omega_{T}}{2}\right)\left[\frac{\omega_{0}^{2}}{\omega_{A}^{2}}-\frac{1}{3}\left(\frac{\delta q^{2}}{q_{0}^{2}}+\beta_{p}^{2} G_{0}\right)\right]=0
$$

Thus, for the drift-tearing branch $\omega_{0}=\omega_{T} / 2$, we find

$$
\Im\left[\delta \omega_{D T}\right]=\frac{2}{\sqrt{\pi}} \frac{v_{\text {the }}}{a} \frac{\beta_{p}^{2} G_{0}\left(B_{0}-A_{0}\right)|\hat{s}|(m+1)}{\frac{1}{3}\left(\delta q^{2} / q_{0}^{2}+\beta_{p}^{2} G_{0}\right)-\frac{\omega_{T}^{2}}{4 \omega_{A}^{2}}} \frac{d_{e}^{2}}{a^{2}},
$$

which is then unstable for $\delta q^{2} / q_{0}^{2}+\beta_{p}^{2} G_{0}>3 \omega_{T}^{2} /\left(4 \omega_{A}^{2}\right)$, when $B_{0}-A_{0}>0$, thus $\delta q<$ $\left(1+|\hat{s}| / 2 q_{0}\right) / n$.

For the MHD branch $\omega_{0}^{2}=\omega_{A}^{2}\left(\delta q^{2} / q_{0}^{2}+\beta_{p}^{2} G_{0}\right) / 3 \equiv \omega_{i n f}^{2}$, and we have

$$
\Im\left[\delta \omega_{M H D}\right]=\frac{2}{\sqrt{\pi}} \frac{\omega_{A}^{2}}{\omega_{0}^{2}} \frac{\beta_{p}^{2} G_{0}\left(B_{0}-A_{0}\right)|\hat{s}|(m+1)}{\frac{\omega_{T}}{2 \omega_{0}}-1} \frac{d_{e}^{2}}{a^{2}} \frac{v_{\text {the }}}{a} .
$$

The MHD branch is then unstable for large enough temperature gradients $3 \omega_{T}^{2} /\left(4 \omega_{A}^{2}\right)>$ $\delta q^{2} / q_{0}^{2}+\beta_{p}^{2} G_{0}$, when $\delta q<\left(1+|\hat{s}| / 2 q_{0}\right) / n$. In our nomenclature, "DT" stands for drifttearing, and "dest. MHD" refers to an MHD mode kinetically destabilized, and not MHD unstable. By using the expression found for $\delta \omega$, one can now check a posteriori that the layer correction on the RHS of Eq. (3.1) is of the same order of the infernal mode drive on the LHS. 
Far from marginality, let us consider $\omega=\omega_{r}+i \gamma$, with $\gamma \gg \omega_{r}$ an take this limit in Eq. (3.9). Then, we find

$$
\gamma_{K I M} \sim\left(\omega_{A}^{2} \frac{v_{t h e}}{a}\right)^{1 / 3} \beta^{2 / 3}\left(\frac{d_{e}}{a}\right)^{2 / 3} \sim n^{0},
$$

and

$$
\omega_{r} \sim \frac{\omega_{T}}{6}<\gamma_{K I M}
$$

Notice that both frequency and growth rate show no scaling with the density, a fact consistent with the phenomenology of the ideal infernal mode shown in the previous section, and with the resistive result (Charlton et al. 1989). If we now take $T_{e}=3.5 \mathrm{keV}$, $T_{i}=2 \mathrm{keV}, n_{0}=10^{13} \mathrm{~cm}^{-3}, a=50 \mathrm{~cm}, B=2.4 \times 10^{4} \mathrm{G}$, then $\beta=3.55 \times 10^{-3} v_{A}=1.72 \times$ $10^{9} \mathrm{~cm} / \mathrm{s}$, with $\omega_{A}=5 v_{A} /(3 a)$, we obtain $v_{\text {the }}=4.19 \times 10^{7} T_{e}^{1 / 2} \mathrm{~cm} / \mathrm{s}=2.45 \times 10^{9} \mathrm{~cm} / \mathrm{s}$, $d_{e}=0.17 \mathrm{~cm}$, which give $\gamma_{K I M} \sim 29 \times 10^{3} \mathrm{sec}^{-1}, \omega_{r} \sim 9 \times 10^{3} \mathrm{sec}^{-1}$. Then, $\gamma_{K I M} / \omega_{r} \sim$ $3>1$, and our approximation is marginally satisfied. From a practical point of view, this result has direct implications in the determination of operation scenarios. Future highperformance plasmas in W7-X will have higher densities than those achieved in presentday experiments. Then, the fact that the growth rate of the instability is insensitive to density would seem good, in the sense that a density increase will not exacerbate the instability. However, the destabilisation mechanism renders such modes virtually unavoidable, if the heating system acts on the electrons increasing their temperature gradient. We must bear in mind, though, that a temperature increase only would not cause instability, this being enabled by the specific shape of the $\iota$-profile. Experimentally, independence on density is a striking physical property that can be investigated via density scans. Scans in $\iota$ can also clarify the role of the excitation of side-band modes that belong to the family of the $(m, n)=(1,1)$ mode.

\section{Conclusions}

In this article we have explored the possibility of obtaining pressure-driven reconnecting modes in devices with a profile of the rotational transform similar to that of the stellarator W7-X. For cylindrical geometries, we have shown, both analytically and numerically, that a nearly flat $\iota$-profile, with an $\iota=1$ surface in the region of small negative magnetic shear, generates unstable modes of the hybrid type between internal pressure-driven kink and infernal. In toroidal axisymmetric geometry, we have extended to kinetic regimes previous work on the viscous-resitive infernal mode. We have found that ion Landau damping has a stabilizing effect; full Larmor radius effects can suppress the infernal mode; the resonance of passing electrons can generate electron-inertia-mediated magnetic reconnection. For a W7-X-like $\iota$-profile, the kinetic resonance can occur at radial locations where side-band harmonics of modes belonging to the family-mode of the $(m, n)=(1,1)$ are excited. Thus, we can argue that the action of such resonant mechanism in the "true" W7-X can cause the instability of the $(m, n)=(1,1)$ mode, due to strong geometric coupling. Kinetic infernal modes are of the diamagnetic and magnetohydrodynamic type, depending on their real frequency. They can be seen as either electron-pressure-driven or current-driven, depending on which branch is considered and how close is $\iota$ to unity in the region where $\iota$ is nearly flat. The condition sufficient for instability is $\delta q=\iota^{-1}-1<\left(1+|\hat{s}| /\left(2 q_{0}\right)\right) / n$. Far from marginality, for typical W7-X parameters, the mode shows a growth rate of tens of kiloHertz, with no explicit scaling on the plasma density. The KIM is an unexpected new ingredient of modern stellarator physics, it is a candidate to explain sawtooth oscillations and poses new challenging questions in the field of optimization of stellarator reactors. 
We thank Matthias Borchardt, Per Helander, Ralf Kleiber, and Marco Zanini for useful discussions on this work.

\section{REFERENCES}

Antonsen, T.M. \& Coppi, Bruno 1981 Non-asymptotic theory of collisionless reconnecting modes. Phys. Letters A $\mathbf{8 1}$ (6), 335 - 338.

Ara, G., Basu, B., Coppi, B., Laval, G., Rosenbluth, M.N. \& Waddell, B.V. 1978 Magnetic reconnection and $\mathrm{m}=1$ oscillations in current carrying plasmas. Annals of Physics 112 (2), 443 - 476.

Brunetti, D, Graves, J P, Cooper, W A \& Wahlberg, C 2014 Fast growing resistive two fluid instabilities in hybrid-like tokamak configuration. Plasma Phys. Control. Fusion 56 (7), 075025.

Brunetti, D, Graves, J P, Halpern, F D, Luciani, J-F, Lütjens, H \& Cooper, W A 2015 Extended MHD simulations of infernal mode dynamics and coupling to tearing modes. Plasma Phys. Control. Fusion 57 (5), 054002.

Charlton, L. A., Hastie, R. J. \& Hender, T. C. 1989 Resistive infernal modes. Phys. Fluids B: Plasma Phys. 1 (4), 798-803.

Connor, J. W., Ham, C. J., Hastie, R. J. \& Zocco, A. 2019 Ion Landau damping and drift tearing modes. Journal Plasma Physics 85 (2), 905850204.

Connor, J W, Hastie, R J \& Zocco, A 2012 Unified theory of the semi-collisional tearing mode and internal kink mode in a hot tokamak: implications for sawtooth modelling. Plasma Physics and Controlled Fusion 54 (3), 035003.

Coppi, B., Mark, J. W. K., Sugiyama, L. \& Bertin, G. 1979 Reconnecting modes in collisonless plasmas. Phys. Rev. Lett. 42, 1058-1061.

Dinklage, A., Beidler, C.D. \& the Wendelstein 7-X team 2018 Magnetic configuration effects on the Wendelstein 7-X stellarator. Nature Phys. 14, 855-860.

Drake, J. F. 1978 Kinetic theory of $\mathrm{m}=1$ internal instabilities. Phys. Fluids 21 (10), 1777-1789.

Glasser, A. H., Greene, J. M. \& Johnson, J. L. 1975 Resistive instabilities in general toroidal plasma configurations. Phys. Fluids 18 (7), 875-888.

Goedbloed, J. P. \& Hagebeuk, H. J. L. 1972 Growth rates of instabilities of a diffuse linear pinch. Phys. Fluids 15 (6), 1090-1101.

Hastie, R.J. \& Hender, T.C. 1988 Toroidal internal kink stability in tokamaks with ultra flat q profiles. Nucl. Fusion 28 (4), 585-594.

Hastie, R. J. 1997 Sawtooth instability in tokamak plasmas. Astrophysics and Space Science 256 (1), 177-204.

Klinger, T, Alonso, A, Bozhenkov, S, Burhenn, R, Dinklage, A, Fuchert, G, Geiger, J, Grulke, O, Langenberg, A, Hirsch, M, Kocsis, G, Knauer, J, Krämer-Flecken, A, Laqua, H, Lazerson, S, Landreman, M, MaAssberg, H, Marsen, S, Otte, M, Pablant, N, Pasch, E, Rahbarnia, K, Stange, T, Szepesi, T, Thomsen, H, Traverso, P, Velasco, J L, Wauters, T, Weir, G, Windisch, T \& The Wendelstein 7-X Team 2017 Performance and properties of the first plasmas of Wendelstein 7-X. Plasma Phys. Control. Fusion 59 (1), 014018.

Klinger, T., Baylard, C., Beidler, C.D., Boscary, J., Bosch, H.S., Dinklage, A., Hartmann, D., Helander, P., Maassberg, M., Peacock, A., Pedersen, T.S., Rummel, T., Schauer, F., Wegener, L. \& Wolf, R. 2013 Towards assembly completion and preparation of experimental campaigns of wendelstein 7-x in the perspective of a path to a stellarator fusion power plant. Fusion Engineering and Design 88 (6), 461-465.

Könies, A. \& Nührenberg, C. 2019 private communication .

Newcomb, William A 1960 Hydromagnetic stability of a diffuse linear pinch. Annals Phys. $10(2), 232-267$.

NührenberG, CARolin 1996 Global ideal magnetohydrodynamic stability analysis for the configurational space of Wendelstein 7-X. Phys. Plasmas 3 (6), 2401-2410.

Nührenberg, J. \& Zille, R. 1987 Equilibrium and stability of low-shear stellarators. Theory of Fusion Plasmas pp. 3-23.

Pegoraro, F., Porcelli, F. \& Schep, T. J. 1989 Internal kink modes in the ion-kinetic regime. Phys. Fluids B: Plasma Phys. 1 (2), 364-374. 
Porcelli, Francesco 1987 Viscous resistive magnetic reconnection. Phys. Fluids 30 (6), 17341742.

Porcelli, F, Boucher, D \& Rosenbluth, M N 1996 Model for the sawtooth period and amplitude. Plasma Phys. Control. Fusion 38 (12), 2163.

Rosenbluth, Marshall N., Dagazian, R. Y. \& Rutherford, P. H. 1973 Nonlinear properties of the internal $\mathrm{m}=1$ kink instability in the cylindrical tokamak. Phys. Fluids $\mathbf{1 6}$ (11), 1894-1902.

Waelbroeck, F. L. \& Hazeltine, R. D. 1988 Stability of low-shear tokamaks. Phys. Fluids 31 (5), 1217-1223.

Zanini, M., Laqua, H.P., Stange, T., Brandt, C., Hirsch, M., Höfel, U., Marushchenko, N., Neuner, U., Rahbarnia, K., Schilling, J., Thomsen, H., Wolf, R.C. \& W7-X TEAM 2019 ECCD operations in the second experimental campaign at W7-X. EPJ Web Conf. 203, 02013.

Zocco, A, Loureiro, N F, Dickinson, D, Numata, R \& Roach, C M 2015 Kinetic microtearing modes and reconnecting modes in strongly magnetised slab plasmas. Plasma Phys. Control. Fusion 57 (6), 065008.

Zocco, Alessandro \& Schekochinin, Alexander A. 2011 Reduced fluid-kinetic equations for low-frequency dynamics, magnetic reconnection, and electron heating in low-beta plasmas. Phys. Plasmas 18 (10), 102309. 